\title{
Removing exogenous information using pedigree data
}

\author{
Carmen Amador • Miguel Ángel Toro • \\ Jesús Fernández
}

\begin{abstract}
Management of certain populations requires the preservation of its pure genetic background. When, for different reasons, undesired alleles are introduced, the original genetic conformation must be recovered. The present study tested, through computer simulations, the power of recovery (the ability for removing the foreign information) from genealogical data. Simulated scenarios comprised different numbers of exogenous individuals taking part of the founder population and different numbers of unmanaged generations before the removal program started. Strategies were based on variables arising from classical pedigree analyses such as founders' contribution and partial coancestry. The efficiency of the different strategies was measured as the proportion of native genetic information remaining in the population. Consequences on the inbreeding and coancestry levels of the population were also evaluated. Minimisation of the exogenous founders' contributions was the most powerful method, removing the largest amount of genetic information in just one generation. However, as a side effect, it led to the highest values of inbreeding. Scenarios with a large amount of initial exogenous alleles (i.e. high percentage of non native founders), or many generations of mixing became very difficult to recover, pointing out the importance of being careful about introgression events in populations where these are undesired.
\end{abstract}

Keywords Undesired introgression - Genetic recovery

\section{Introduction}

The benefits of interchanging genetic resources between populations have been deeply studied. New inputs of genetic material are usually considered a good way to protect species and biological diversity from threats such as the lost of ability to adapt to new environments and the decrease in fitness (Frankham et al. 2002). However, gene flow can lead to a undesired introduction of genetic material into the population. Apart from the problems which could arise from outbreeding depression (Allendorf and Luikart 2007) sometimes there is a need of maintaining the population genetic background pure. In that case, when an undesired introduction of genetic material happens, the original genetic conformation would have to be recovered.

There are numerous examples where keeping the original background would be interesting. In the field of domestic animals, a particular breed could be linked to a quality product with economical interest. Many breeds provide differentiated products directly related to the specific genetic information of the breed: Iberian pigs and the Jamón Ibérico, Reggiana dairy cows and the Parmigiano Reggiano cheese and some other examples pointed by Dalvit et al. (2007) There are also specific breeds connected to a particular activity, as the Spanish and other purebred horses which are involved in sport competitions and other entertainment events with high economical benefits (MAPA 2003). Just for aesthetical reasons dog breeds are kept separated. A dog can become a official member of a breed only if both parents belong to it, being economically devaluated when it is a mixed-breed (Parker et al. 2004). The loss of value of crossed individuals is also common in other species. 
From a conservationist point of view, gene flow can also be harmful for some animal and plant species or populations due to the replacement of native populations by invaders. Some examples of admixture and introgression have been described in birds (quail, Barilani et al. 2005; partridges, Negro et al. 2001), fishes (grayling, Sušnik et al. 2004; trout, Boyer et al. 2008), bovine (cattle, Padilla et al. 2009; bison, Freese et al. 2007; Halbert and Derr 2007) and carnivores (wolves, Miller et al. 2003; cats, Beaumont et al. 2001).

Livestock breeds are considered important components of biodiversity, and its conservation is based on pure-breeding (Hall and Bradley 1995). Human activities contribute to increase rates of hybridization and introgression. The relevance of this problem has been underestimated, leading in the worst case scenario to populations going to extinction. This phenomenon is happening more frequently than expected (Rhymer and Simberloff 1996; Allendorf et al. 2001). In farm animals, microsatellite markers have revealed the occurrence of introgression (Groeneveld et al. 2010).

Another scenario where undesired introgression occurs is when facing the task of reconstructing a completely or partially extinct breed by using cryoconserved semen on females from another breed or species (Gandini and Oldenbroek 2007). The $50 \%$ of the genome coming from the semen bank would be from the original background, but the other $50 \%$ coming from the females would be undesired and would have to be eliminated to achieve the complete recovery of the original species.

A good example of undesired introgression (in this case due to an incorrect management of the population) is the Przewalski's horse (Bouman and Bouman 1994). Przewalski's horse (Equus przewalskii) is an endangered species that was almost extinct after the World War II. It was described in 1881 and its entire genealogy has been kept since 1899. In the Zoological Department of the Agricultural Institute of the University of Halle in Germany, counsellor J. Kühn crossed a Przewalski's horse with a Mongolian domestic horse producing one colt (1906) that had more descendants with another Przewalski's. Domestic horse alleles introgressed the pure population and they still remain in the present Przewalski's horses. As the entire genealogy is kept and available (Volf 1994) the expected percentage of foreign or pure genome of each individual can be calculated.

When the introgression process takes place, the crossed individuals can be detected or not by visual signs. When the admixture is not reflected morphologically, undesired information could be removed using recorded pedigree information, if available, or using markers that allow detecting the alleles coming from different populations.

The objective of the present study is to explore the efficiency of different methods to remove the exogenous genetic information from an introgressed population using exclusively pedigree information.

\section{Methods}

Simulated data

A population with size $(N)$ of 10 individuals ( 5 males and 5 females), kept constant over discrete generations was simulated. The pedigree was recorded all generations since the beginning, and used during the management. The genome of each individual was made up of 20 chromosomes of $1 \mathrm{M}$ each. A total of 100 multiallelic loci were simulated per chromosome. A Poisson distributed number $(\lambda=1)$ of crossingovers with no interference were generated in random positions of each chromosome when creating the offspring.

In the initial generation individuals were not inbred, and unrelated. They carried two different alleles at each locus ( $2 N$ different alleles per locus in the base population) and, thus, loci were completely informative. This molecular information was used to calculate different parameters at the end of the management period to evaluate the efficiency of the methods.

Different scenarios with different degrees of introgression were simulated by varying the following factors:

Number of exogenous individuals: The percentage of introgression ranged from 10 to $50 \%$ by including $1-5$ exogenous individuals (sex randomly set) as part of the base population. Native and exogenous individuals carried different alleles in all the 100 loci.

Number of generations without management: A number (1-5) of initial unmanaged generations (random contributions and mating) were simulated to obtain the admixture of the foreign information that was set in the base population and to create some relatedness among individuals.

\section{Management}

After the initial unmanaged phase, 10 generations of management were performed. Four different strategies were carried out in order to determinate contributions of individuals to the next generation (i.e. offspring generated by each potential parent):

(1) Random (R): The individuals mated randomly and parents were not selected.

(2) Optimum contributions (OC): optimal contributions from parents were calculated by minimising the global coancestry weighted by those contributions,

$$
\sum_{i=1}^{N} \sum_{j=1}^{N} c_{i} c_{j} f_{i j},
$$

where $c_{i}$ is the relative contribution of individual $i$ to the next generation, and $f_{i j}$ is the coancestry between individuals $i$ and $j$ (Caballero and Toro 2000). Strategy R corresponds to unmanaged populations and, thus, it provides the 
lower bound for the desintrogression process. Contrarily, $\mathrm{OC}$ (the standard management procedure recommended for conservation programmes) is directed to control the loss of genetic diversity and the rise of inbreeding. Therefore, both methods can be used as reference points for evaluating the performance of other strategies.

(3) Minimum exogenous contribution (MEC): the contributions were obtained by minimising the total amount of information which came from the exogenous founders,

$$
\sum_{i=1}^{N} c_{i} f_{E x, i}
$$

where $f_{E x, i}$ is the coancestry between individual $i$ and all the exogenous founders.

(4) Minimum partial coancestry (MPC): contributions were calculated by minimizing the mean partial coancestry,

$$
\sum_{i=1}^{N} \sum_{i=1}^{N} c_{i} c_{j} f_{i j}^{*},
$$

where $f_{i j} *$ is the partial coancestry between $i$ and $j$. The partial coancestry represents the kinship between two individuals due to one specific ancestor, or, in other terms, it is the probability that an offspring from that couple is homozygous for an allele descending from a specific ancestor. Sum of partial coancestries due to each of the founders is the total coancestry. Partial coancestry can be determined by using a modification of the tabular method (Lacy et al. 1996; Lacy 1997). Under MPC strategy, partial coancestries included in the objective function were those due to the foreigner founders. Therefore, the sum across all the exogenous founders provided the value for partial coancestry used to compare individuals and to decide their contributions.

MEC and MPC methods were also implemented in all scenarios adding a restriction on the maximum rate of inbreeding $(\Delta F)$. Three different restrictions were imposed: 5,10 and $15 \%$. Also extra simulations were carried out with a population size of 100 individuals with $10,20,30$, 40 or 50 exogenous individuals in the base population, followed by up to 5 generations without management and 10 generations under the four treatments.

All the optimizations were performed using simulated annealing algorithms (Kirkpatrick et al. 1983; Fernandez and Toro 1999). Once the optimum contributions were obtained, minimum coancestry matings were performed in all scenarios, except for the random management, by implementing the Hungarian algorithm (Dantzig 1963). For each scenario and management method 20 replicates were simulated and results presented are averages over replicates.

\section{Variables}

In each generation several variables were calculated to evaluate the efficiency of the different strategies: (1) nonexogenous founder representation, calculated from genealogy, (2) non-exogenous founder representation, calculated from genomic molecular information, (3) average inbreeding coefficient, (4) mean coancestry, (5) mean partial coancestry and (6) observed homozygosity. Values for 3, 4 and 5 were calculated from pedigree.

Real data

The conditions of the captive breeding programme of the Przewalski's horse have allowed keeping the complete pedigree of the horse since 1899 , which is available in http://przwhorse.pikeelectronic.com/. The genealogy was analyzed and the descendents of the Mongolian domestic mare introduced in 1906 were detected. All individuals not descending from the foreign introgressed horse were identified and the relationship between them and the whole population was studied from 1935 to 2009 to evaluate the potential of desintrogression at different times. The consequences of removing carriers of exogenous alleles, both on the levels of genetic diversity and inbreeding coefficient, were also evaluated.

\section{Results}

\section{Simulated data}

Non-exogenous founder genealogical representation

Results for non-exogenous founder representation are shown in Fig. 1. As expected, the $\mathrm{R}$ and $\mathrm{OC}$ strategies did not eliminate any exogenous representation but kept the values constant irrespective of the number of generations elapsed before management started, as only drift is affecting the frequency of foreign alleles. Note that native information observed at generation 10 under these strategies is near $90,80,70,60$ and $50 \%$ of that present in the base population for 1, 2, 3, 4 and 5 exogenous founders, respectively.

The most effective method for removing undesired introgression was always MEC (Fig. 1). Maximum values of non-exogenous founder representation were always obtained when using this strategy.

In some cases, MPC could reach the efficiency of MEC, particularly when the numbers of foreigners and nonmanaged generations were low. Differences between both methods became larger as these parameters increased. When the percentage of introgression was high or many generations elapsed till the management, the chance for 


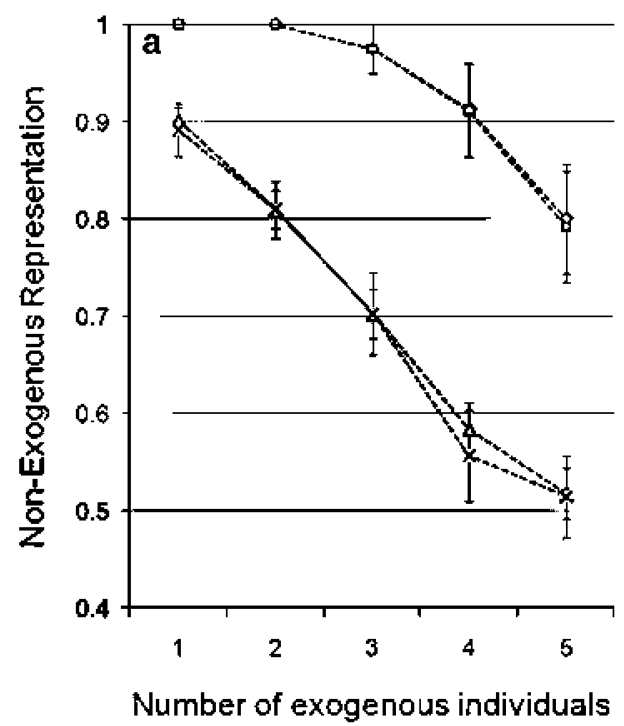

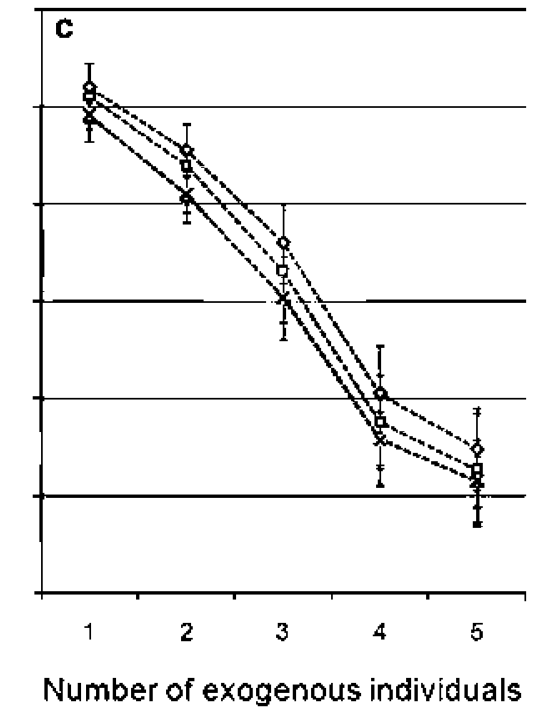

Number of exogenous individuals
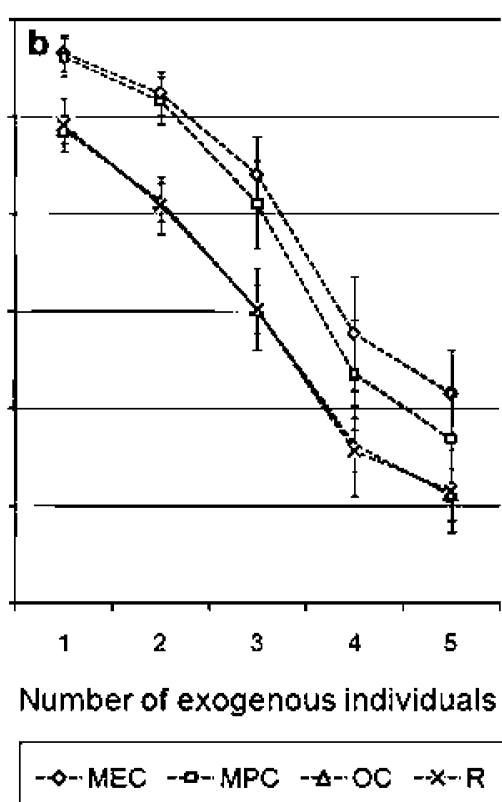

Fig. 1 Non-exogenous representation in the last generation of management with each of the four methods, according to the number of exogenous individuals. a One previous generation with no

introgressed information being removed became too low irrespective of the strategy used (see right part of Fig. 1c). Only in the scenarios with a small number of foreigners and a low number of generations of admixture, the original genomic information will be completely recovered.

It must be pointed out that the final value of the nonexogenous founder representation was reached in the first generation of management, and did not change afterwards under both MEC and MPC strategies (data not shown). This fact indicates that it would be enough one generation of management under MEC to obtain the best result.

Results about foreign representation calculated from the molecular information of each individual's genome follow the same pattern as the observed for the genealogical information in all cases (data not shown). This happened because the simulated loci are neutral, unlinked and completely informative in the base population, and thus genealogical and molecular coancestries are equivalent.

\section{Inbreeding coefficient}

The performance of both inbreeding and mean coancestry was similar. Consequently only the evolution of the inbreeding coefficient $(F)$ is presented in Fig. 2. The minimum inbreeding coefficient was obtained, as expected, with the OC method. MEC always led to the maximum values of inbreeding and mean coancestry even above the $\mathrm{R}$ method.

Under the $\mathrm{R}$ and $\mathrm{OC}$ strategies, the inbreeding levels reached were independent of the number of exogenous management, b Three previous non-managed generations, c Five previous non-managed generations. Vertical bars are standard errors of the estimates

individuals in the base population but increased with the number of unmanaged initial generations. Contrarily, the number of exogenous founders affected the levels of inbreeding in MEC and MPC.

MPC led to similar results as MEC in scenarios with a low number of unmanaged generations and/or with a low number of exogenous founders (i.e. with little admixture). As the number of exogenous individuals increased the results of $\mathrm{MPC}$ differed from those of $\mathrm{MEC}$ and became more similar to those of $\mathrm{OC}$. This performance is due to the fact that the greater is the number of exogenous, more founders are to be taken into account when calculating the partial coancestry and, therefore, approaching the global coancestry.

The unpleasant performance of $\mathrm{MEC}$ regarding the levels of inbreeding or coancestry is a consequence of the importance that this strategy gives, by definition, to the elimination of foreign information, ignoring global genetic diversity. Thus, all individuals with the same percentage of their genomes coming from exogenous founders are equivalent, and $\mathrm{MEC}$ assigns the same value to solutions where they contribute equally, differentially or even when some do not contribute at all. Moreover, when foreign information is evenly distributed among individuals (i.e. exogenous representation is equal in all individuals) MEC turns into Random management, as the method lacks a criterion to prioritise individuals.

To alleviate this effect a modification of the method was implemented. It consisted in selecting from all the 


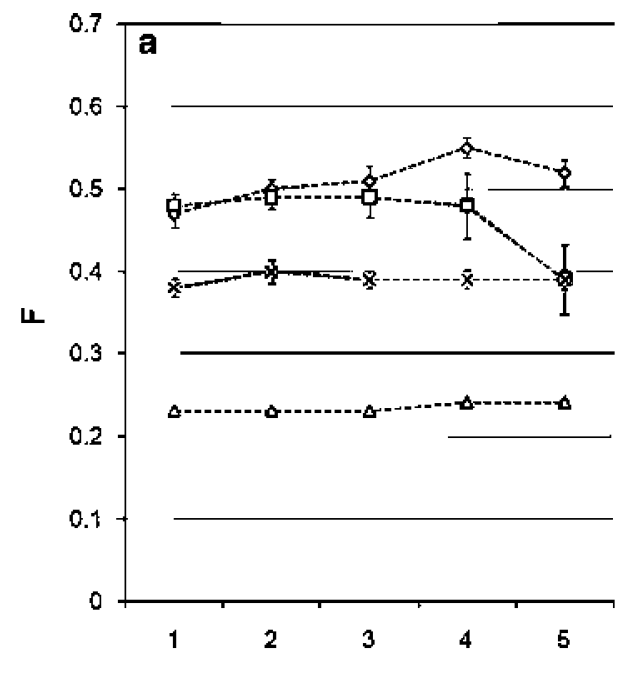

Number of exogenous individuals

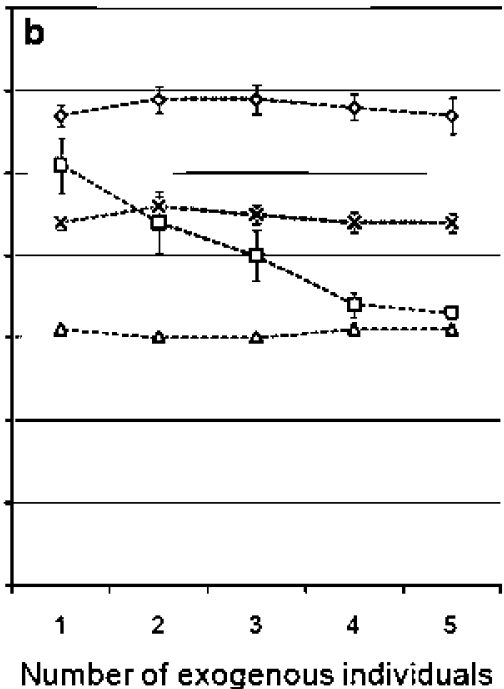

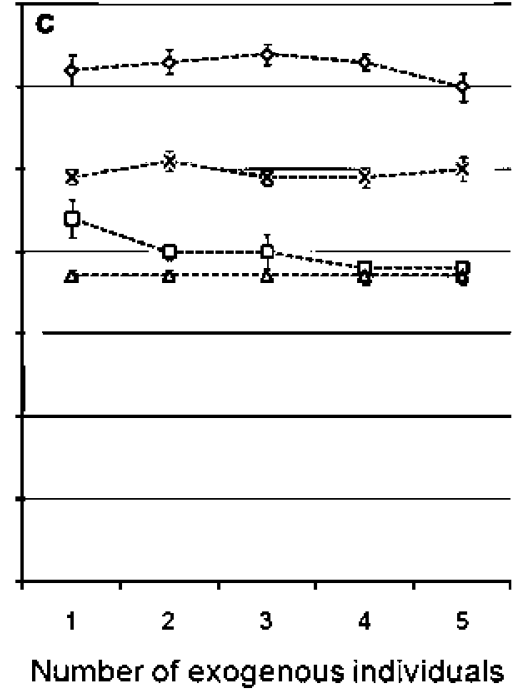

Number of exogenous individuals
Fig. 2 Inbreeding coefficient in the last generation of management with each of the four methods, according to the number of exogenous individuals. a One previous generation with no management, $\mathbf{b}$ Three previous non-managed generations, c Five previous non-managed generations. Vertical bars are standard errors of the estimates

\section{Partial coancestry}

solutions with the same remaining proportion of exogenous information the one with the maximum number of individuals contributing to the next generation. This improved method yielded slightly lower levels of inbreeding, although still greater than the other methods (data not shown).

Another trend in the performance of the inbreeding coefficient under MEC could be observed. Cases with an intermediate number of foreigners showed larger values of $F$ and mean coancestry. This could be explained by the fact that, when there was little introgression, most available individuals to be used as parents for the next generation are completely free of exogenous influence and, thus, it is not necessary to reduce the number of used parents to perform the desintrogression. In cases with many exogenous founders, most individuals have undesired introgression, but they are almost equivalent and, consequently, all used as parents (there is no removal, but the genetic diversity is maintained). However, with an intermediate number of foreign founders just a few "pure" individuals (i.e. without exogenous ancestors) remain to be used as parents and, similar to a bottleneck effect, the mean inbreeding and coancestry increase.

When simulations using MEC and MPC included restrictions on the increase of inbreeding $(5,10$ and $15 \%$ of rate of inbreeding per generation) results were very similar to those obtained under OC method irrespective of the restriction imposed. While coancestry and inbreeding were kept low, there was no removal of exogenous representation in any of the performed scenarios (data not shown).
Results of the evolution of partial coancestry are shown in Fig. 3. As expected, the minimum value of partial coancestry was always obtained by minimising the partial coancestry (MPC), since this strategy was developed to do so. Populations under the R strategy yielded a partial coancestry value which increased with the number of exogenous individuals, and it was always larger than that with OC. As it happened with inbreeding, MPC became similar to OC when the number of foreigners (and also the number of unmanaged generations) was large. This is due to partial coancestry representing a part of the kinship that it is greater the larger is the number of foreigners founding the population.

When the number of unmanaged generations or external individuals was small, MEC led to a lower partial coancestry than $\mathrm{R}$ and $\mathrm{OC}$, and to similar partial coancestry than MPC. As soon as the number of unmanaged generations or exogenous founders increased, the efficiency of MEC in keeping low levels of partial coancestry decreased. That could be explained by the fact that the individuals, despite they had lower exogenous percentage of information, were more related through the remaining foreign lineages. It has to be taken into account that the minimisation of the partial coancestry is not a specific objective of the MEC method.

\section{Observed homozygosity}

The performance of the observed homozygosity calculated from the simulated loci in all methods is equivalent to the 


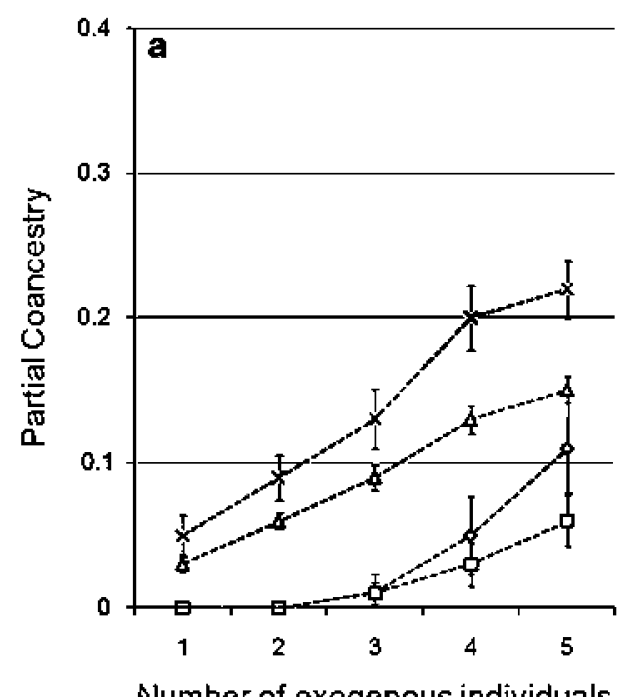

Number of exogenous individuals

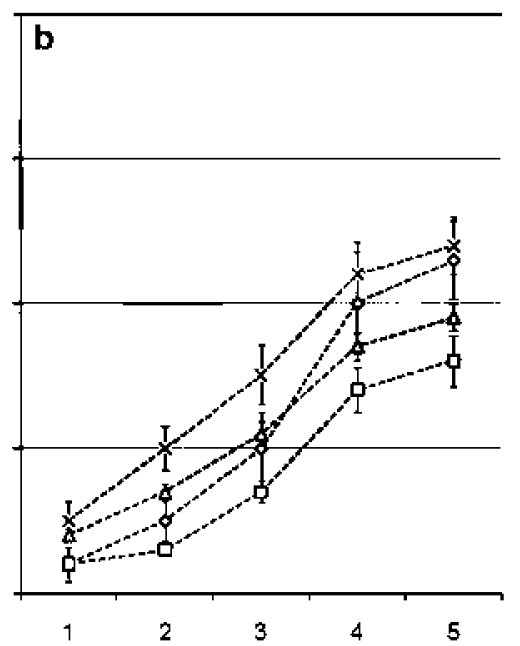

Number of exogenous individuals

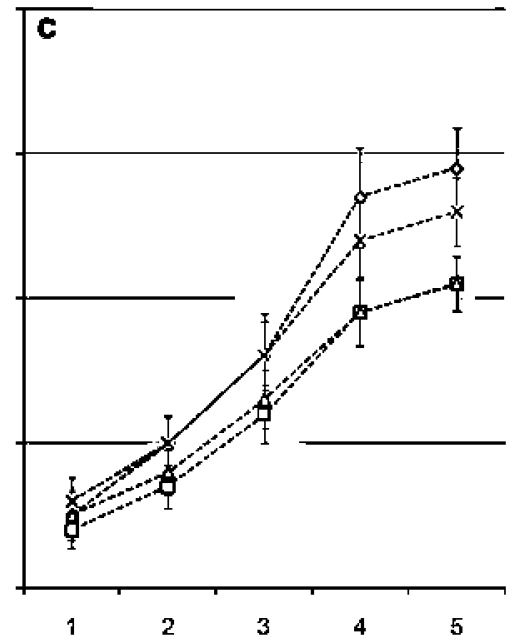

Number of exogenous individuals

$-\infty$ MEC -口-MPC - -OC --x-R

Fig. 3 Partial coancestry in the last generation of management with each of the four methods, according to the number of exogenous individuals. a One previous generation with no management, b Three

pedigree inbreeding coefficient. Its value is not genealogical but represents the realisation at the genomic level, and represents the identity by descent because the 2000 loci of each individual are neutral and completely informative in the base population.

Large population Table 1 shows the non-exogenous founder genealogical representation and the inbreeding coefficient achieved after ten generations of management for MEC and MPC strategies, $N=10$ and $N=100$ individuals, and a level of introgression from 10 to $50 \%$. Results for $\mathrm{R}$ and $\mathrm{OC}$ were similar to the those obtained under these strategies in the 10 individuals population, with no desintrogression and $F$ levels only dependent on the number of unmanaged generations (data not shown). Comparisons between the two population sizes showed that the efficiency of both methods increased with a larger number of individuals for the same percentage of introgression. In all cases previous non-managed generations, c Five previous non-managed generations. Vertical bars are standard errors of the estimates

lower levels of exogenous information were achieved in the 100 individuals population, and, of course, with a lower increase of inbreeding. The MEC strategy proved to be better than the MPC strategy especially with a large degree of introgression. MPC performed similarly to MEC when the percentage of exogenous founders was $10 \%$ (with similar values of native representation and $F$ ) but it got worse with more than $20 \%$ of exogenous individuals.

As in the scenario with $N=10$, the whole effect of MEC on the removal of foreign information in populations with 100 individuals was achieved in the first generation of management. This did not happen when managing with MPC where several generations were needed to remove the undesired information, inducing, thus, an increase in inbreeding. In this case, the efficiency of MEC is higher because the information is removed quickly and the increase of inbreeding is lower in the first generation.

Table 1 Comparison of non-exogenous representation and inbreeding coefficient after ten generations of management (under MEC and MPC) on populations of size $N=10$ and $N=100$ individuals, with $10-50 \%$ of introgression and 5 generations of admixture

Non-exogenous representation

\begin{tabular}{lrlllll}
\hline \multicolumn{7}{c}{ Level of introgression } \\
\cline { 3 - 7 } & \multicolumn{1}{rl}{$N$} & $10 \%$ & $20 \%$ & $30 \%$ & $40 \%$ & $50 \%$ \\
\hline MEC & 10 & 0.920 & 0.855 & 0.760 & 0.605 & 0.548 \\
& 100 & 0.990 & 0.921 & 0.876 & 0.766 & 0.676 \\
MPC & 10 & 0.911 & 0.839 & 0.731 & 0.576 & 0.527 \\
& 100 & 0.984 & 0.864 & 0.784 & 0.649 & 0.537 \\
\hline
\end{tabular}

F

\begin{tabular}{lrlllll}
\hline \multicolumn{7}{c}{ Level of introgression } \\
\cline { 3 - 7 } & \multicolumn{1}{c}{$N$} & $10 \%$ & $20 \%$ & $30 \%$ & $40 \%$ & $50 \%$ \\
\hline \multirow{2}{*}{ MEC } & 10 & 0.615 & 0.634 & 0.644 & 0.632 & 0.599 \\
& 100 & 0.167 & 0.240 & 0.264 & 0.258 & 0.271 \\
MPC & 10 & 0.435 & 0.401 & 0.399 & 0.379 & 0.376 \\
& 100 & 0.159 & 0.066 & 0.051 & 0.043 & 0.041 \\
\hline
\end{tabular}


Real data

Results from the Przewalski's horse studbook analysis are shown in Table 2 which gives the total number of reproductive individuals with their correspondent mean coancestry $(f)$ as well as the number of reproductive individuals with no relationship with the undesired mare (i.e. individuals with no introgressed information) with their global $f$ from 1935 to 2010 . The total number of Przewalski's horse has quickly increased since 1980 , but the influence of the Mongolian domestic horse introduced is still maintained, as no particular management strategy has been implemented to remove it.

From a total of 1,800 theoretical reproductive individuals currently alive, just 182 are no related to the introgressed mare. The $f$ of this group of individuals is twice the $f$ of the total population, reflecting a huge reduction of the genetic diversity harboured by the pure subset. Currently, just a small part of the population remains pure and with a high inbreeding level. But similar levels are found when looking at the beginning of the recorded genealogy. The influence of the exogenous horse was quickly spread into the population so just a little percentage of highly related individuals were not descendant of the foreign mare when a few generations since the introgression elapsed.

\section{Discussion}

Gene flow between populations is usually considered beneficial because it can protect the biological diversity and increases fitness by avoiding the rise of inbreeding depression. Some population analysis point out that extremely endangered populations can only be restored by introgressing new genetic material, looking for an increase in fitness due to the reduction of inbreeding that introducing exogenous alleles can produce. This process is called genetic rescue. It has been proved that very low levels of

Table 2 Results of the Przewalski's horse analysis

\begin{tabular}{lrrrrr}
\hline Year & \multicolumn{3}{c}{ Total } & & \multicolumn{2}{c}{ Native } \\
\cline { 2 - 3 } & $N_{\text {ind }}$ & $f$ & & $N_{\text {ind }}$ & $f$ \\
\hline 1935 & 38 & 0.053 & 26 & 0.093 \\
1940 & 32 & 0.075 & 22 & 0.120 \\
1950 & 23 & 0.084 & 13 & 0.132 \\
1960 & 35 & 0.154 & 17 & 0.234 \\
1970 & 100 & 0.175 & 42 & 0.246 \\
1980 & 201 & 0.155 & 65 & 0.266 \\
1990 & 506 & 0.145 & 111 & 0.269 \\
2000 & 1023 & 0.144 & 129 & 0.254 \\
2010 & 1800 & 0.140 & 182 & 0.257 \\
\hline
\end{tabular}

migration are enough to recover most of the genetic variation lost in a small population. (Ingvarsson 2001; Tallmon et al. 2004).

However, some disadvantages of the admixture of genetic information have been also pointed out, when dealing with wild or domestic species, such as economical devaluation or replacement of native populations by invaders. When undesirable introgression has occurred there is a need of developing methodologies to remove the exogenous genetic information in order to recover the original background related to the economic interest or the biodiversity component. In the present study, methods based in genealogical information were tested for their accuracy in the depuration, and the effects on other genetic measures of the population, mainly $F$ and $f$, were determined.

For the whole range of parameters evaluated in the simulations, it was proved that the best strategy to remove undesired information is to avoid the contribution to the next generations of those carrying the highest proportion of genetic information coming from the exogenous founders. The MEC method achieved the best results regarding the elimination of exogenous alleles in all scenarios studied. Results were the same when measuring genealogical or genomic representation of the native founders.

Notwithstanding, the power of the strategy is limited by the total number of individuals and foreigners in the population, as well as the number of generations of mixing. However, even considering a long period of introgression, when the population is large enough the ability of finding individuals completely unrelated to the exogenous founders become higher. Therefore, the size of the population is also a very important factor that affects the possibility of recovery, and obviously, the levels of inbreeding reached after the management.

The principle of the MEC method implies to select among the available candidates those individuals which keep the highest percentage of native background (ideally without foreign information). Therefore, it reduces considerably the number of animals contributing to the next generation and provokes the large increase of inbreeding reflected in Fig. 2 (similar results were observed in observed homozygosity for the genomic data). The high level of coancestry induced by the MEC strategy denotes a large loss of diversity, being this phenomenon a drawback of the method. Actions taken to avoid this side effect (i.e. a restriction in the increase of the inbreeding coefficient) led MEC to perform like $\mathrm{OC}$ with slow increases of $F$, but also with no removal of undesired information. It is clear, then, that the level of desintrogression (and the speed of the process) is directly related to this increase in inbreeding. Less stringent restrictions on the acceptable $\Delta F$ can be tested to look for an equilibrium between the degree of removal and the loss of diversity. 
As mentioned above, the removal of introgression under MEC is accomplished in the first generation of management. The method selects among all the available individuals, those with less exogenous influence. When these individuals mate, they produce all the descendants with the exact same proportion of relationship with the exogenous founders and, therefore, in the next generation MEC cannot choose among them for a second generation of management. If at least one male and one female can be found unrelated to all exogenous founders these individuals will be selected and all descendant from that moment will be also unrelated to foreigners. Consequently, no possibility of desintrogression will exist in later generations as all candidates would be equally valuable. This redistribution of the exogenous genetic information makes the method ineffective after just one generation. Thus, after implementing $\mathrm{MEC}$ in the first generation in order to achieve the maximization of the native genetic background, the sensibly strategy would be to keep on managing the population with OC strategy to minimise the further increase in $F$ and the loss of genetic diversity.

General results point out the importance of the time passed away between the introgression and the starting of the management. They show that with few generations without management, a small amount of introgression can spread into the population and turn out almost impossible to recover. Therefore, it is very important to act as soon as possible to keep introgression controlled. Results of the simulations are confirmed with real data from the Przewalski's horse Studbook, where the influence of a single foreigner greatly influences the whole pedigree and remains noticeable in the current population, 100 years later. The important efforts made in the past to save the Przewalski's horse and the current programs to reintroduce it in wild have been very successful in achieving the preservation of a species that was almost extinct (less than 50 horses after the World War II). Currently it is a potentially viable population living in the border between Mongolia and China (Bouman and Bouman 1994). However, after 100 years, the influence of the Mongolian domestic horse introduced in the population is still visible and the efforts to remove it would imply a highly loss of viability making the idea of recover original Przewalski's horse background unfeasible.

The concern about conserving populations pure, especially livestock breeds (Hall and Bradley 1995), lead us to warn about the drawbacks of admixture. The results of this study enhance the importance of keeping recorded genealogies of the populations, and show the importance of a quick reaction against introgression to keep it under control. The efficiency of the information provided by genealogies depends on the total amount of individuals and the percentage of undesired introgression and admixture.
The genealogy has to be completely available to be useful to our desintrogression purposes, which could be relatively common in livestock breeds, but not feasible in natural populations threatened for other invasive species or breeds where keeping track of individuals is difficult or impractical (Allendorf and Luikart 2007). In this case, there is a need to incorporate extra information. Molecular information can provide a tool to identify the breed to which animal belongs to. As molecular markers were capable of reveal introgression (Groeneveld et al. 2010), they will be useful for accomplishing the opposite effect, i.e. helping in the identification of the undesired individuals that are more related to the exogenous genetic background. Molecular markers can also be helpful for reconstructing the pedigree (Butler et al. 2004) allowing to apply the strategy suggested in this study. Also the molecular coancestry can be used to trace those individuals which have a foreign origin and select among the population individuals less related to them.

The conclusion from the present study is that even small undesired introgression can lead a population to quickly get mixed and lose the genetic conformation that it is intended to be preserved. In that case actions should be taken as soon as possible to recover most of the original genetic background. The importance of keeping pedigree records, or the possibility of reconstructing it through molecular markers, was also pointed out.

Acknowledgments This work was financed by Plan Nacional I+D+i (Spain) CGL2009-13278-C02-02. Carmen Amador was supported by a FPI-INIA Grant (INIA/Ministerio de Ciencia e Innovación). We are thankful to Luis Alberto García Cortés for making available the whole pedigree of Przewalski's horse and to Beatriz Villanueva on useful comments on the manuscript.

\section{References}

Allendorf FW, Luikart G (2007) Conservation and the genetics of populations. Blackwell, Malden

Allendorf FW, Leary RF, Spruell P, Wenburg JK (2001) The problems with hybrids: setting conservation guidelines. Trends Ecol Evol 16:613-622

Barilani M, Deregnaucourt S, Gallego S, Galli L, Mucci N, Piombo R, Puigcerver M, Rimondi S, Rodriguez-Teijeiro JD, Spano S, Randi E (2005) Detecting hybridization in wild (Coturnix $c$. coturnix) and domesticated (Coturnix c. japonica) quail populations. Biol Conserv 126:445-455

Beaumont M, Barratt EM, Gottelli D, Kitchener AC, Daniels MJ, Pritchard JK, Bruford MW (2001) Genetic diversity and introgression in the Scottish wildcat. Mol Ecol 10:319-336

Bouman I, Bouman J (1994) The history of the Przewalski's horse. In: Boyd L, Houpt KA (eds) Przewalski's horse: the history and biology of an endangered species. State University of New York Press, New York, pp 5-38

Boyer MC, Muhlfeld CC, Allendorf FW (2008) Rainbow trout (Oncorhynchus mykiss) invasion and the spread of hybridization with native westslope cutthroat trout (Oncorhynchus clarkii lewisi). Can J Fish Aquat Sci 65:658-669 
Butler K, Field C, Herbinger CM, Smith BR (2004) Accuracy, efficiency and robustness of four algorithms allowing full sibship reconstruction from DNA marker data. Mol Ecol 13:1589-1600

Caballero A, Toro MA (2000) Interrelations between effective population size and other pedigree tools for the management of conserved populations. Genet Res 75:331-343

Dalvit C, De Marchi M, Cassandro M (2007) Genetic traceability of livestock products: a review. Meat Sci 77:437-449

Dantzig GB (1963) Linear programming and extensions. Princeton University Press, Princeton

Fernandez J, Toro MA (1999) The use of mathematical programming to control inbreeding in selection schemes. J Anim Breed Genet 116:447-466

Frankham R, Ballou JD, Briscoe DA (2002) Introduction to conservation genetics. Cambridge University Press, Cambridge

Freese CH, Aune KE, Boyd DP, Derr JN, Forrest SC, Gates CC, Goyan PJP, Grassel SM, Halbert ND, Kunkel K, Redford KH (2007) Second chance for the plains bison. Biol Conserv 136: $175-184$

Gandini G, Oldenbroek K (2007) Strategies for moving from conservation to utilisation. In: Oldenbroek K (ed) Utilisation and conservation of farm animal genetic resources. Wageningen Academic Publishers, Wageningen, pp 29-54

Groeneveld LF, Lenstra JA, Eding H, Toro MA, Scherf B, Pilling D, Negrini R, Finlay EK, Jianlin H, Groeneveld E, Weigend S, Consortium G (2010) Genetic diversity in farm animals-a review. Anim Genet 41:6-31

Halbert ND, Derr JN (2007) A comprehensive evaluation of cattle introgression into US federal bison herds. J Hered 98:1-12

Hall SJG, Bradley DG (1995) Conserving livestock breed biodiversity. Trends Ecol Evol 10:267-270

Ingvarsson PK (2001) Restoration of genetic variation lost-the genetic rescue hypothesis. Trends Ecol Evol 16:62-63

Kirkpatrick S, Gelatt CD, Vecchi MP (1983) Optimization by simulated annealing. Science 220:671-680
Lacy RC (1997) Errata. Evolution 51:1025

Lacy RC, Alaks G, Walsh A (1996) Hierarchical analysis of inbreeding depression in Peromyscus polionotus. Evolution 50: $2187-2200$

MAPA, (de) Ministerio de Agricultura, Pesca y Alimentación. (2003) Estudio y caracterización del sector equino en España, Madrid. http://www.eurocarne.com/informes/pdf/sector-equino.pdf

Miller CR, Adams JR, Waits LP (2003) Pedigree-based assignment tests for reversing coyote (Canis latrans) introgression into the wild red wolf (Canis rufus) population. Mol Ecol 12:3287-3301

Negro JJ, Torres MJ, Godoy JA (2001) RAPD analysis for detection and eradication of hybrid partridges (Alectoris rufa $\times$ A. graeca) in Spain. Biol Conserv 98:19-24

Padilla JA, Sansinforiano E, Parejo JC, Rabasco A, Martínez-Trancón M (2009) Inference of admixture in the endangered Blanca Cacereña bovine breed by microsatellite analyses. Livest Sci $122: 314-322$

Parker HG, Kim LV, Sutter NB, Carlson S, Lorentzen TD, Malek TB, Johnson GS, DeFrance HB, Ostrander EA, Kruglyak L (2004) Genetic structure of the purebred domestic dog. Science 304:1160-1164

Rhymer JM, Simberloff D (1996) Extinction by hybridization and introgression. Annu Rev Ecol Syst 27:83-109

Sušnik S, Berrebi P, Dovc P, Hansen MM, Snoj A (2004) Genetic introgression between wild and stocked salmonids and the prospects for using molecular markers in population rehabilitation: the case of the Adriatic grayling (Thymallus thymallus $\mathbf{L}$. 1785). Heredity 93:273-282

Tallmon DA, Luikart G, Waples RS (2004) The alluring simplicity and complex reality of genetic rescue. Trends Ecol Evol 19:489-496

Volf J (1994) The Studbook. In: Boyd L, Houpt KA (eds) Przewalski's horse: the history and biology of an endangered species. State University of New York Press, New York, pp 61-73 